\title{
Pharmacokinetics and Exposure-Response Analyses of Daratumumab in Combination Therapy Regimens for Patients with Multiple Myeloma
}

\author{
Xu Steven Xu • Meletios A. Dimopoulos • Pieter Sonneveld · P. Joy Ho · Andrew Belch • \\ Merav Leiba · Marcelo Capra · David Gomez · Eva Medvedova · Shinsuke Iida · Chang-Ki Min · \\ Jordan Schecter · Richard Jansson · Liping Zhang · Yu-Nien Sun · Pamela L. Clemens
}

Received: August 20, 2018 / Published online: October 29, 2018

(C) The Author(s) 2018

\begin{abstract}
Introduction: Daratumumab, a human IgG monoclonal antibody targeting CD38, has demonstrated activity as monotherapy and in combination with standard-of-care regimens in multiple myeloma. Population pharmacokinetic analyses were conducted to determine the pharmacokinetics of intravenous daratumumab in combination therapy versus monotherapy, evaluate the effect of patient- and disease-
\end{abstract}

Enhanced digital features To view enhanced digital features for this article go to https://doi.org/10.6084/ m9.figshare.7165493.

Electronic supplementary material The online version of this article (https://doi.org/10.1007/s12325018-0815-9) contains supplementary material, which is available to authorized users.

X. S. Xu $(\bowtie) \cdot J$. Schecter

Janssen Research \& Development, LLC, Raritan, NJ, USA

e-mail:sxu26@its.jnj.com

M. A. Dimopoulos

National and Kapodistrian University of Athens,

Athens, Greece

P. Sonneveld

Department of Hematology, Erasmus MC,

Rotterdam, The Netherlands

P. J. Ho

Royal Prince Alfred Hospital, Camperdown,

New South Wales, Australia related covariates on drug disposition, and examine the relationships between daratumumab exposure and efficacy/safety outcomes. Methods: Four clinical studies of daratumumab in combination with lenalidomide/dexamethasone (POLLUX and GEN503); bortezomib/dexamethasone (CASTOR); pomalidomide/ dexamethasone, bortezomib/thalidomide/dexamethasone, and bortezomib/melphalan/prednisone (EQUULEUS) were included in the analysis. Using various dosing schedules, the majority of patients $(684 / 694)$ received daratumumab at a dose of $16 \mathrm{mg} / \mathrm{kg}$. In GEN503, daratumumab was administered at a dose of $2 \mathrm{mg} / \mathrm{kg} \quad(n=3), \quad 4 \mathrm{mg} / \mathrm{kg} \quad(n=3), \quad 8 \mathrm{mg} / \mathrm{kg}$ $(n=4)$, and $16 \mathrm{mg} / \mathrm{kg} \quad(n=34)$. A total of 650 patients in EQUULEUS $(n=128)$, POLLUX $(n=282)$, and CASTOR $(n=240)$ received daratumumab $16 \mathrm{mg} / \mathrm{kg}$. The exposure-efficacy
A. Belch
Cross Cancer Institute, Edmonton, AB, Canada
M. Leiba
Assuta Ashdod University Hospital, Faculty of Health Sciences, Ben Gurion University of the Negev, Beersheba, Israel
M. Capra
Instituto do Cancer, Hospital Mãe de Deus,
Porto Alegre, Brazil
D. Gomez
Hospital Universitario de la UANL, Monterrey, Nuevo Leon, Mexico 
and exposure-safety relationships examined progression-free survival (PFS) and selected adverse events (infusion-related reactions; thrombocytopenia, anemia, neutropenia, lymphopenia, and infections), respectively.

Results: Pharmacokinetic profiles of daratumumab were similar between monotherapy and combination therapy. Covariate analysis identified no clinically important effects on daratumumab exposure, and no dose adjustments were recommended on the basis of these factors. Maximal clinical benefit on PFS was achieved for the majority of patients (approximately $75 \%$ ) at the $16 \mathrm{mg} / \mathrm{kg}$ dose. No apparent relationship was observed between daratumumab exposure and selected adverse events.

Conclusion: These data support the recommended $16 \mathrm{mg} / \mathrm{kg}$ dose of daratumumab and the respective dosing schedules in the POLLUX and CASTOR pivotal studies.

Funding: Janssen Research \& Development.

Keywords: CD38; Daratumumab; Pharmacokinetics; Multiple myeloma; Oncology

\section{INTRODUCTION}

Despite recent advancements in treatment strategies for multiple myeloma (MM), outcomes remain poor and treatment options are limited for patients who relapse following therapy with a proteasome inhibitor (PI) and an immunomodulatory drug (IMiD), or those who are refractory to these agents [1, 2]. Daratumumab is a human monoclonal antibody that targets CD38 and induces anti-myeloma activity through on-tumor and immunomodulatory

E. Medvedova

Oregon Health \& Science University, Portland, OR, USA

S. Iida

Nagoya City University Hospital, Nagoya, Japan

C.-K. Min

Seoul St. Mary's Hospital, Seoul, South Korea

R. Jansson · L. Zhang · Y.-N. Sun · P. L. Clemens Janssen Research \& Development, LLC, Spring House, PA, USA mechanisms of action [3-8]. Clinical studies of daratumumab monotherapy (GEN501 and SIRIUS) demonstrated deep and durable responses in patients with heavily treated relapsed or relapsed and refractory MM [9, 10]. Clinical and pharmacokinetics (PK) data from these studies led to the approval of daratumumab monotherapy in many countries worldwide [11] and established the recommended dose $(16 \mathrm{mg} / \mathrm{kg}$ actual body weight) and dosing schedule (once weekly for 8 weeks, every 2 weeks for 16 weeks, and every 4 weeks thereafter) for treatment [10, 12-14]. In a pooled analysis of GEN501 and SIRIUS, daratumumab monotherapy $(16 \mathrm{mg} / \mathrm{kg})$ was well tolerated, and patients with heavily treated MM achieved an overall response rate (ORR) of $31.1 \%[10,15]$.

Recent studies have indicated that there is a significant benefit to patient outcomes when daratumumab is combined with various background therapy regimens. The combination of daratumumab with lenalidomide and dexamethasone (D-Rd) resulted in an ORR of $81 \%$ in the single-arm phase 1/2 GEN503 study of the combination in heavily treated patients with refractory or relapsed and refractory MM [16]. In the phase 3 POLLUX study, D-Rd elicited a significant $63 \%$ reduction in disease progression or death compared with lenalidomide and dexamethasone (Rd) alone [17]. Similarly, in the phase 3 CASTOR study, daratumumab plus bortezomib and dexamethasone (D-Vd) significantly reduced the risk of progression or death by $61 \%$ compared to bortezomib and dexamethasone ( $\mathrm{Vd}$ ) alone in patients with relapsed and refractory disease treated with a median of two lines of therapy [18]. On the basis of these pivotal studies, daratumumab in combination with Rd or Vd was first approved in the USA and Europe [19], and subsequently many other countries, for the treatment of patients with $\mathrm{MM}$ who have received one or more prior lines of therapy. A recent phase 1 study (EQUULEUS) of daratumumab in combination with pomalidomide and dexamethasone (D-Pd) yielded promising results, with an ORR of $60 \%$, and $29 \%$ of patients who achieved a complete response or better reached minimal residual disease negative status at a sensitivity threshold of $10^{-5}$ [20]. On the basis of these findings, D-Pd 
was approved in the USA for patients with MM who have received at least two prior lines of therapy including lenalidomide and a proteasome inhibitor. Recently, daratumumab added to the standard of care regimen of bortezomib, melphalan, and prednisone (D-VMP) was shown to reduce the risk of progression or death by $50 \%$ in transplant-ineligible newly diagnosed MM patients [21].

PK data from the monotherapy studies (GEN501 and SIRIUS) indicate that daratumumab exhibits nonlinear PK consistent with target-mediated drug disposition [13]. Population PK modeling showed that efficacious concentrations of daratumumab were achieved rapidly in patients receiving daratumumab $16 \mathrm{mg} / \mathrm{kg}$ via the standard dosing schedule and were maintained as the frequency of dosing was reduced $[12,14]$. Given the promising efficacy of daratumumab when combined with other treatments across all lines of treatment, it is necessary to understand the PK of daratumumab when administered as part of a combination regimen and the relationship of daratumumab exposure with efficacy and safety.

The purpose of this investigation was to determine the PK of daratumumab in combination therapy. Population PK analyses were performed using data from four clinical studies of daratumumab combination therapy including POLLUX, GEN503, CASTOR, and EQUULEUS. We compared $\mathrm{PK}$ of combination therapies versus daratumumab monotherapy, evaluated the effect of patient- and disease-related covariates on drug disposition, and examined the relationships between daratumumab exposure and safety and efficacy outcomes.

\section{METHODS}

\section{Patients and Study Designs}

Population PK analyses were performed on combined datasets from four clinical studies: CASTOR (MMY3004; ClinicalTrials.gov identifier, NCT02136134), POLLUX (MMY3003; NCT02076009), GEN503 (NCT01615029), and EQUULEUS (MMY1001; NCT01998971), all of which have been described in detail elsewhere $[16-18,20]$. Key eligibility criteria for each study are shown in Supplemental Table 1. CASTOR was a randomized, active-controlled, multicenter, phase 3 study comparing D-Vd to Vd alone in patients with relapsed or relapsed and refractory MM [18]. In CASTOR, patients received daratumumab $16 \mathrm{mg} / \mathrm{kg}$ once weekly for 9 weeks (cycles 1-3), every 3 weeks for 15 weeks (cycles 4-8), and every 4 weeks thereafter (Supplemental Table 2). POLLUX was a randomized, active-controlled, multicenter, phase 3 study comparing D-Rd to Rd alone in patients with relapsed or refractory MM [17]. In POLLUX, daratumumab $16 \mathrm{mg} / \mathrm{kg}$ was administered once weekly for 8 weeks (cycles 1-2), then every 2 weeks for 16 weeks (cycles 3-6), and then every 4 weeks thereafter. GEN503 was a phase $1 / 2$, open-label, dose-escalation (part 1) and dose-expansion (part 2) study of D-Rd in patients with refractory or relapsed and refractory MM [16]. In part 2 of GEN503, patients received daratumumab $16 \mathrm{mg} / \mathrm{kg}$ on the same dosing schedule as the POLLUX study. EQUULEUS was a multi-arm, phase $1 / 2$, open-label study of daratumumab in combination with a variety of backbone regimens in newly diagnosed and relapsed or refractory MM [20]. Dosing schedules were once weekly for 6 weeks [cycles 1-2 for Vd and bortezomib, thalidomide, and dexamethasone (VTd); cycle 1 for bortezomib, melphalan, and prednisone (VMP)], then every 3 weeks thereafter, or were consistent with the dosing schedule in POLLUX (Rd), depending on the combination partners.

In the combined analysis, daratumumab was administered intravenously to a total of 694 patients using various dosing schedules. A total of 650 patients in EQUULEUS $(n=128)$, POLLUX $(n=282)$, and CASTOR $(n=240)$ received daratumumab $16 \mathrm{mg} / \mathrm{kg}$. In GEN503, a total of 34 patients received daratumumab $16 \mathrm{mg} / \mathrm{kg}$, while the remaining patients received daratumumab at doses of $2 \mathrm{mg} / \mathrm{kg}$ $(n=3), 4 \mathrm{mg} / \mathrm{kg}(n=3)$, and $8 \mathrm{mg} / \mathrm{kg}(n=4)$. Thus, the population PK dataset included 694 patients, 684 of whom received daratumumab $16 \mathrm{mg} / \mathrm{kg}$. Patients who received less than $16 \mathrm{mg} / \mathrm{kg}$ of daratumumab were not included in the efficacy and safety analysis. 


\section{Compliance with Ethics Guidelines}

All studies were conducted in accordance with the International Conference on Harmonisation Good Clinical Practice guidelines and the principles of the Declaration of Helsinki, and all patients provided written informed consent.

\section{Population PK and Exposure-Response Analysis}

Analyses were performed on pooled datasets from CASTOR, POLLUX, GEN503, and EQUULEUS. In CASTOR, samples were collected within $2 \mathrm{~h}$ prior to and immediately following daratumumab infusions on day 1 of cycles 1,3 , 6, 9, and 12 (cycles 1-8: 21 days; cycles 9+: 28 days). In POLLUX, samples were collected within $2 \mathrm{~h}$ prior to and immediately following daratumumab infusions on day 1 of cycles 1,3 , and 12 (28-day cycles). In both CASTOR and POLLUX, samples were collected at the end of treatment and during the follow-up visit. In GEN503, part 1, samples were collected prior to and at the end of infusion on all daratumumab infusion days, at each follow-up visit, and at the end of treatment visit. In part 2 of GEN503, the sampling schedule was reduced to day 1 of cycles $1,3,6$, and 12 , and at weeks 4 and 8 after the last treatment dose. In EQUUELUS, samples from the D-VMP and D-Pd regimens were collected within $2 \mathrm{~h}$ prior to and at the end of infusion on days 1 and 22 of cycle 1 , day 1 of cycles $2-4$, and weeks 3 and 9 of the follow-up phase (D-VMP: 42-day cycles; D-Pd: 28-day cycles). In the D-VTd and D-Vd arms of EQUUELUS, samples were collected within $2 \mathrm{~h}$ prior to and at the end of infusion on day 1 of cycles 1-4 and at weeks 3 and 9 of the follow-up phase (D-VTd and D-Vd: 21-day cycles). Serum daratumumab concentrations were evaluated using a validated enzymelinked immunosorbent assay [lower limit of quantitation (LLOQ) $=0.2 \mu \mathrm{g} / \mathrm{mL}$; BioAnalytical Research Corporation Global Central Laboratory, Ghent, Belgium; Janssen Research \& Development, LLC, Spring House, PA, USA). Immunogenicity was analyzed by determining the presence of anti-daratumumab antibodies. A population PK model, previously developed using data from daratumumab monotherapy studies [14], was used to fit concentration-time data from the four combination studies. No direct impact of the background therapies on the PK of daratumumab was expected given the lack of overlapping clearance mechanisms for daratumumab and the co-administered smallmolecule therapies [22-24]. NONMEM $^{\circledR} 7.2$ software (ICON, Dublin, Ireland) was used for population PK modeling. Software package R (version 3.1.2) was used for data management, post-processing, and all other analyses of NONMEM runs. PK simulations were run using the dosing schedules for CASTOR and for POLLUX.

Subgroup analyses were performed to evaluate the relationship between daratumumab exposure and patient- and disease-related characteristics, including age, race, sex, body weight, hepatic or renal impairment, type of myeloma (immunoglobulin [Ig] G versus nonIgG), baseline Eastern Cooperative Oncology Group status, number of prior therapies, and refractory status. The relationship between daratumumab exposure [maximal pre-infusion (trough) concentration $\left.\left(C_{\text {pre-infusion,max }}\right)\right]$ and progression-free survival (PFS) was examined for the POLLUX and CASTOR studies in which PFS was the primary endpoint. The relationship between $C_{\text {pre-infusion,max }}$ and adverse events (AEs) of interest, selected because of their frequency in anti-myeloma treatment (i.e., incidence of thrombocytopenia, anemia, neutropenia, lymphopenia, and infections), was examined by treatment regimen [i.e., D-Rd (combined data from POLLUX and GEN503), D-Vd (CASTOR), and D-Pd (EQUULEUS)]. To evaluate the relationship between the incidence of infusion-related reactions (IRRs) and daratumumab exposure, the predicted end-of-infusion concentration after the first infusion $\left(C_{\max , 1 s t}\right)$ was used because the majority of IRRs occurred during the first infusion.

Cox proportional hazard regression models, implemented in the "survival" package in the software program $\mathrm{R}$, were used to explore the relationship between $C_{\text {pre-infusion,max }}$ and PFS for CASTOR and POLLUX using P-splines. The respective control groups were used as a reference level for calculating relative hazard. The exposure-safety relationship was explored on the 
basis of exposure quantiles of $C_{\text {post-infusion,max }}$ and their corresponding 95\% confidence interval for the selected AEs and $C_{\text {max,1st }}$ for IRRs.

\section{RESULTS}

\section{Patient and Disease Characteristics}

The population PK dataset included 4426 measurable samples from 694 patients (POLLUX, $n=282$; GEN503, $n=44$; EQUULEUS, $n=128$; CASTOR, $n=240$ ), 684 of whom received daratumumab $16 \mathrm{mg} / \mathrm{kg}$. A small proportion (2.5\%) of the samples were below the LLOQ of $0.2 \mu \mathrm{g} / \mathrm{mL}$ for daratumumab and excluded from the population PK analysis. Descriptive statistics of baseline continuous and categorical patient and disease covariates are summarized in Supplemental Table 3 and Supplemental Table 4, respectively.

\section{Comparison of PK of Daratumumab Following Monotherapy and Combination Therapy Regimens}

Regardless of the background regimen in the treatment combination, daratumumab concentrations were similar to those in the monotherapy studies (Supplemental Fig. 1). The similar PK of daratumumab between the monotherapy and combination therapy studies suggested that the small-molecule combinations would not impact daratumumab PK. As in the monotherapy studies, concentration-time data for daratumumab in combination regimens were adequately described by a two-compartment population PK model with parallel linear and non-linear Michaelis-Menten eliminations. The parameter estimates of the final covariate model are presented in Supplemental Table 5. Exposure to daratumumab was similar between monotherapy and combination therapies. The average predicted $C_{\text {pre-infusion,max }}$ was $632.8 \mu \mathrm{g} / \mathrm{mL}$ following combination therapy with daratumumab using the POLLUX dosing schedule (after 8 weekly infusions) and $667.0 \mu \mathrm{g} / \mathrm{mL}$ for the CASTOR schedule (after 9 weekly infusions) versus $530.7 \mu \mathrm{g} / \mathrm{mL}$ following daratumumab monotherapy (after 8 weekly infusions). The model-derived mean $[ \pm$ standard deviation (SD)] half-life associated with linear elimination was $23.3 \pm 11.8$ days in the combination studies, assuming the standard (POLLUX-like) dosing schedule, compared with $18.0 \pm 9.0$ days in the monotherapy studies. Consistent with the monotherapy studies, steady state levels of daratumumab were
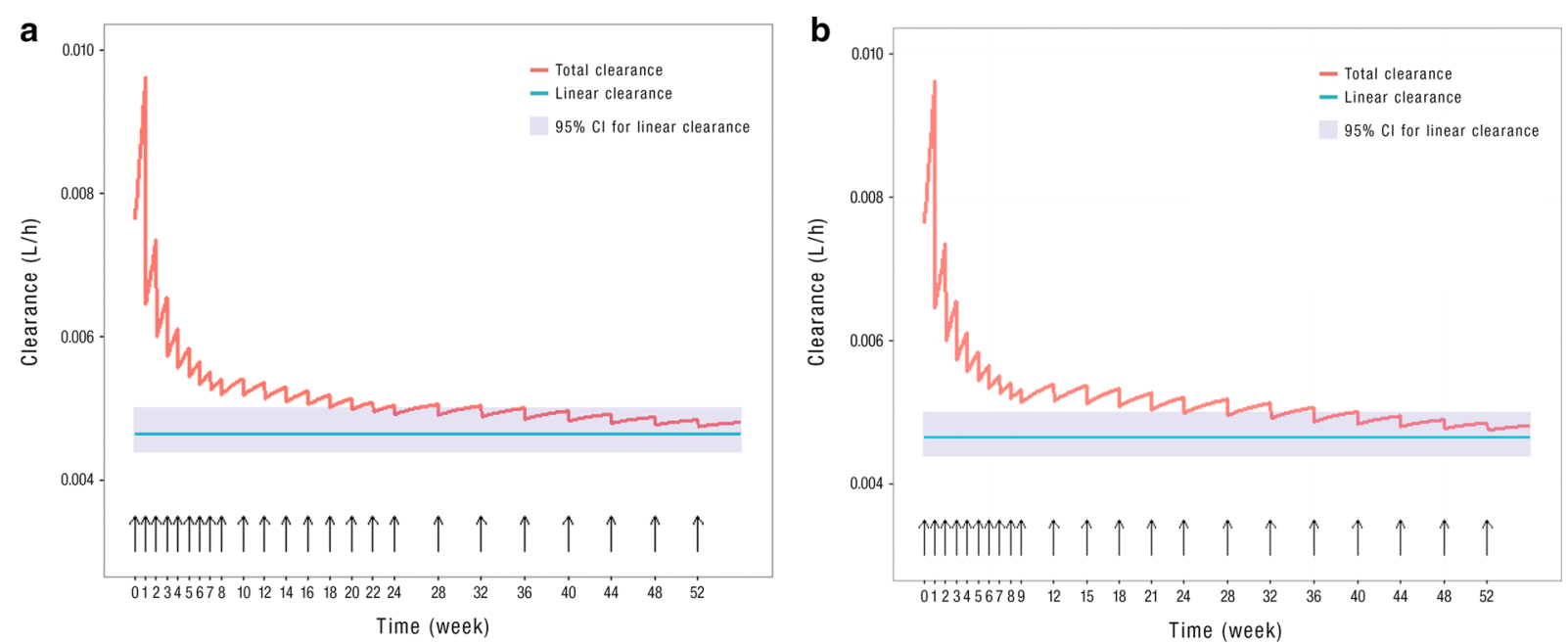

Fig. 1 Model-based simulation of daratumumab clearance versus time using POLLUX (a) and CASTOR (b) dosing schedules. The red line represents total clearance, and the blue line represents linear clearance. The blue shaded region delineates the $95 \%$ CI for linear clearance. Arrows represent dosing events. CI confidence interval 

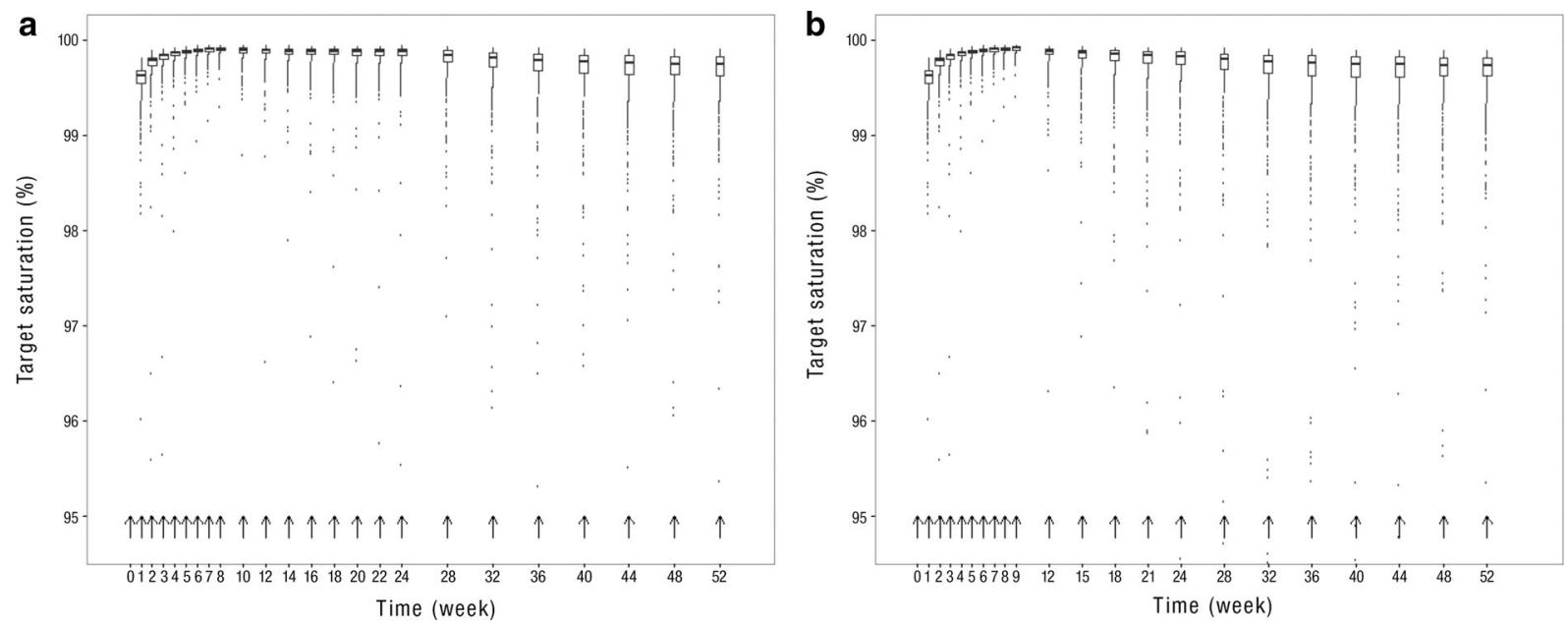

Fig. 2 Target saturation profile of daratumumab at pre-infusion time points for the POLLUX (a) and CASTOR (b) dosing schedules. For the POLLUX dosing schedule (a), the simulations were performed assuming the dosing schedule of QW for 8 weeks, Q2W for 16 weeks, and then Q4W thereafter. For the CASTOR dosing schedule (b), the simulations were performed assuming the dosing schedule of QW for 9 weeks, Q3W for 15 weeks, and then Q4W for 32 weeks thereafter. The predicted target saturation was calculated as $100 \times C /\left(K_{\mathrm{M}}+C\right)$, where $C$ represents the pre-infusion (trough) concentration at each time point. Arrows represent dosing events. $Q W$ weekly, $Q 2 W$ every 2 weeks, $Q 4 W$ every 4 weeks, $Q 3 W$ every 3 weeks

reached at approximately 5 months into the every-4-week (Q4W) dosing period. The total clearance of daratumumab decreased over time and approached the non-specific linear clearance after approximately 8 weeks under the standard dosing schedule (Fig. 1a) as well as the different dosing schedule used in the CASTOR study (Fig. 1b). Total clearance continued to stay close to linear clearance during the Q4W dosing period, suggesting that target saturation was maintained. During the Q4W dosing period, concentrations consistent with 99\% target saturation were maintained at trough concentrations in more than $90 \%$ of patients treated with the recommended dosing schedule (Fig. 2a) and the CASTOR dosing schedule (Fig. 2b).

\section{Effects of Patient and Disease Characteristics on Daratumumab Exposure}

The effects of patient and disease characteristics on the estimated $C_{\text {pre-infusion,max }}$ were similar to or smaller than those estimated using the model based on the monotherapy studies [14] (Table 1) and were similar regardless of dosing schedule (data not shown). Consistent with the results from the monotherapy studies, all of the investigated covariate effects were within $25 \%$ of the reference value. Clearance and volume of distribution of daratumumab increased with increasing body weight; however, daratumumab exposure differed by less than $20 \%$ across patients with varied body weights when daratumumab was administered on a milligram per kilogram basis. Daratumumab exposure was approximately $23 \%$ lower in patients with IgGtype MM compared with those who had nonIgG MM. No dose adjustments are recommended on the basis of the presence of the IgG myeloma subtype [14].

\section{Relationship Between Daratumumab Exposure and Efficacy}

An exposure-response analysis on PFS for POLLUX and CASTOR was done with their control groups ( $\mathrm{Rd}$ and $\mathrm{Vd}$, respectively) as the reference level to calculate the relative hazard (Fig. 3a, b). The relative hazard for PFS and 
Table 1 Effect of covariates on daratumumab exposure: comparison of change relative to reference value of the $C_{\text {pre-infusion,max }}$ between monotherapy and combination therapies (POLLUX dosing schedule)

\begin{tabular}{|c|c|c|}
\hline Covariate & Monotherapy & Combination therapies \\
\hline \multicolumn{3}{|l|}{ Renal function } \\
\hline Mild vs. normal & $1.15(0.86-1.54)$ & $0.99(0.91-1.07)$ \\
\hline Moderate vs. normal & $0.96(0.71-1.30)$ & $0.97(0.89-1.06)$ \\
\hline Severe vs. normal & $1.00(0.47-2.12)$ & $1.02(0.78-1.32)$ \\
\hline \multicolumn{3}{|l|}{ Hepatic function } \\
\hline Mild vs. normal & $0.71(0.51-0.98)$ & $1.01(0.91-1.12)$ \\
\hline Moderate/severe vs. normal & $\mathrm{NE}$ & $0.95(0.63-1.41)$ \\
\hline \multicolumn{3}{|l|}{ Age, years } \\
\hline$\geq 65$ vs. $<65$ & $1.07(0.84-1.35)$ & $1.06(0.99-1.13)$ \\
\hline$\geq 75$ vs. $<75$ & $1.09(0.71-1.69)$ & $1.01(0.90-1.13)$ \\
\hline \multicolumn{3}{|l|}{ Sex } \\
\hline Male vs. female & $0.86(0.68-1.09)$ & $0.96(0.90-1.03)$ \\
\hline \multicolumn{3}{|l|}{ Race } \\
\hline White vs. non-white & $0.88(0.61-1.27)$ & $1.10(1.01-1.19)$ \\
\hline \multicolumn{3}{|l|}{ Body weight ${ }^{\mathrm{a}}$, $\mathrm{kg}$} \\
\hline Q3 vs. Q4 & $1.06(0.76-1.47)$ & $0.98(0.90-1.08)$ \\
\hline Q2 vs. Q4 & $0.90(0.65-1.25)$ & $0.89(0.81-0.98)$ \\
\hline Q1 vs. Q4 & $0.76(0.54-1.06)$ & $0.81(0.74-0.90)$ \\
\hline \multicolumn{3}{|l|}{ Albumin, g/L } \\
\hline$<35$ vs. $\geq 35($ normal $)$ & $0.72(0.57-0.92)$ & $0.79(0.74-0.86)$ \\
\hline \multicolumn{3}{|l|}{ Prior line of therapy } \\
\hline 2 vs. 1 & NA & $0.98(0.90-1.06)$ \\
\hline 3 vs. 1 & NA & $0.97(0.88-1.07)$ \\
\hline$>3$ vs. 1 & NA & $0.84(0.75-0.93)$ \\
\hline$>3$ vs. $\leq 3$ & $0.83(0.63-1.11)$ & NA \\
\hline \multicolumn{3}{|l|}{ Refractory status } \\
\hline PI only vs. none & NA & $0.93(0.82-1.06)$ \\
\hline IMiD only vs. none & NA & $0.95(0.84-1.08)$ \\
\hline Double vs. none & NA & $0.92(0.81-1.05)$ \\
\hline Double vs. other & $1.08(0.80-1.46)$ & NA \\
\hline \multicolumn{3}{|l|}{ ECOG status } \\
\hline 1 vs. 0 & $0.88(0.68-1.15)$ & $0.99(0.92-1.06)$ \\
\hline
\end{tabular}


Table 1 continued

\begin{tabular}{llc}
\hline Covariate & Monotherapy & Combination therapies \\
\hline 2 vs. 0 & $0.85(0.51-1.43)$ & $0.95(0.82-1.11)$ \\
Type of myeloma & & $0.77(0.73-0.83)$ \\
IgG vs. non-IgG & $0.50(0.40-0.62)$ & 0.77 \\
\hline
\end{tabular}

The $C_{\text {pre-infusion,max }}$ for the monotherapy and combination therapies were $530.65 \mu \mathrm{g} / \mathrm{mL}(494.6-566.7)$ and $632.8 \mu \mathrm{g} / \mathrm{mL}$ (620.0-645.6), respectively

$N E$ not evaluable, $Q$ quantile, $N A$ not applicable because of different grouping for monotherapy and combination therapy analyses, $P I$ proteasome inhibitor, IMiD immunomodulatory drug, ECOG Eastern Cooperative Oncology Group, Ig immunoglobulin

${ }^{a}$ The quantiles of body weight for combination studies were Q1 $<64.6 \mathrm{~kg}, \mathrm{Q} 2>64.6$ to $\leq 75.9 \mathrm{~kg}, \mathrm{Q} 3>75.9$ to $\leq 88.0 \mathrm{~kg}$, and Q4 $>88.0 \mathrm{~kg}$. The quantiles of body weight for monotherapy studies were Q1<63.9 kg, Q2 $>63.9$ to $\leq 78.6 \mathrm{~kg}, \mathrm{Q} 3>78.6$ to $\leq 88.1 \mathrm{~kg}$, and $\mathrm{Q} 4>88.1 \mathrm{~kg}$

depth of response decreased rapidly with increasing daratumumab exposure based on the data from POLLUX and CASTOR (data not shown). When the maximum trough concentration was approximately $250 \mu \mathrm{g} / \mathrm{mL}$, the risk compared to the control group was substantially reduced (by approximately $25 \%$ for POLLUX and CASTOR). When $C_{\text {pre-infusion,max }}$ was

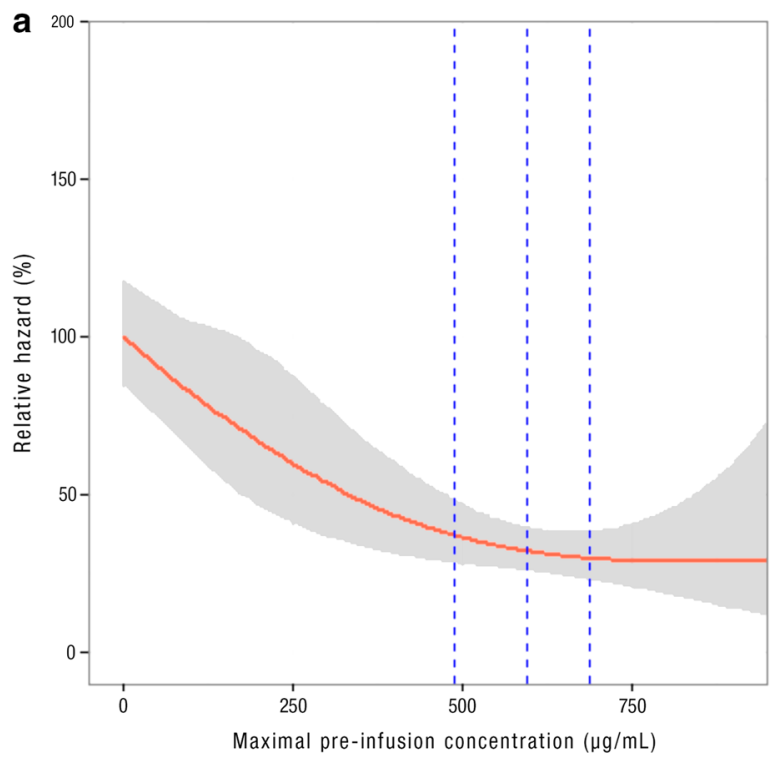

greater than $250 \mu \mathrm{g} / \mathrm{mL}$, the decline in relative hazards appeared to slow down, suggesting limited additional benefit at higher pre-infusion daratumumab concentrations. The majority of patients (approximately 75\%) approached maximum effect, indicating that the maximum clinical benefit on PFS was achieved at the recommended $16 \mathrm{mg} / \mathrm{kg}$ dose and dosing schedule.

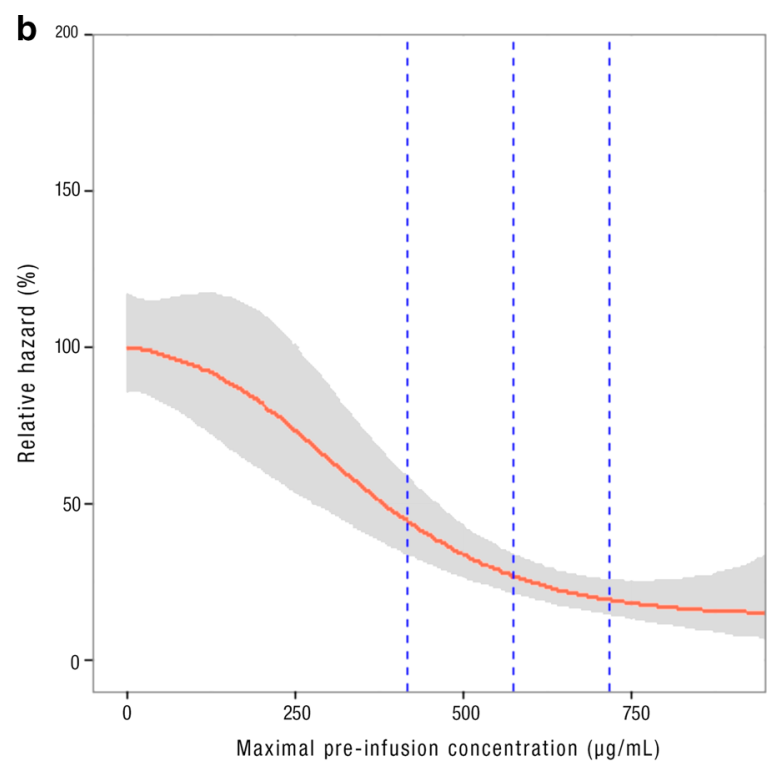

Fig. 3 Relative hazard of PFS at different predicted maximal trough concentrations for POLLUX (a) and CASTOR (b). The solid red line is the point estimate, and the gray shaded areas represent the $95 \%$ CI. The blue vertical dotted lines separate the quartiles of $C_{\text {pre-infusion,max }}$. The control group of each study (Rd in POLLUX and Vd in CASTOR) was used as the reference (i.e., $C_{\text {pre-infusion,max }}=0$ ). $C_{\text {pre-infusion,max }}$ up to the 8 th QW dose for POLLUX and CASTOR. Stratified Cox regression models based on risk stratification of the patients were used to estimate the relative hazard. PFS progression-free survival, $C I$ confidence interval, $R d$ lenalidomide and dexamethasone, $V d$ bortezomib and dexamethasone, $Q W$ weekly 


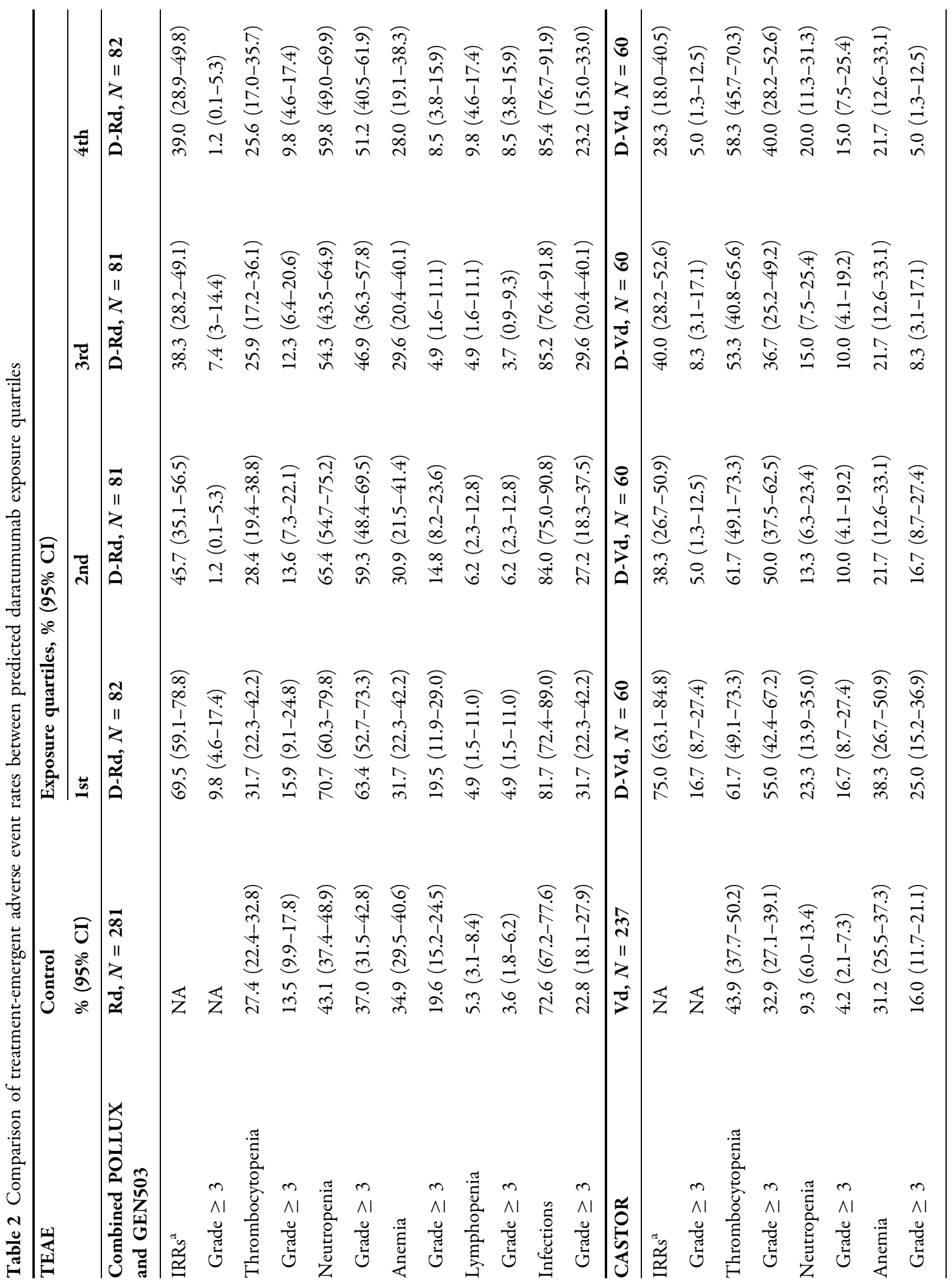




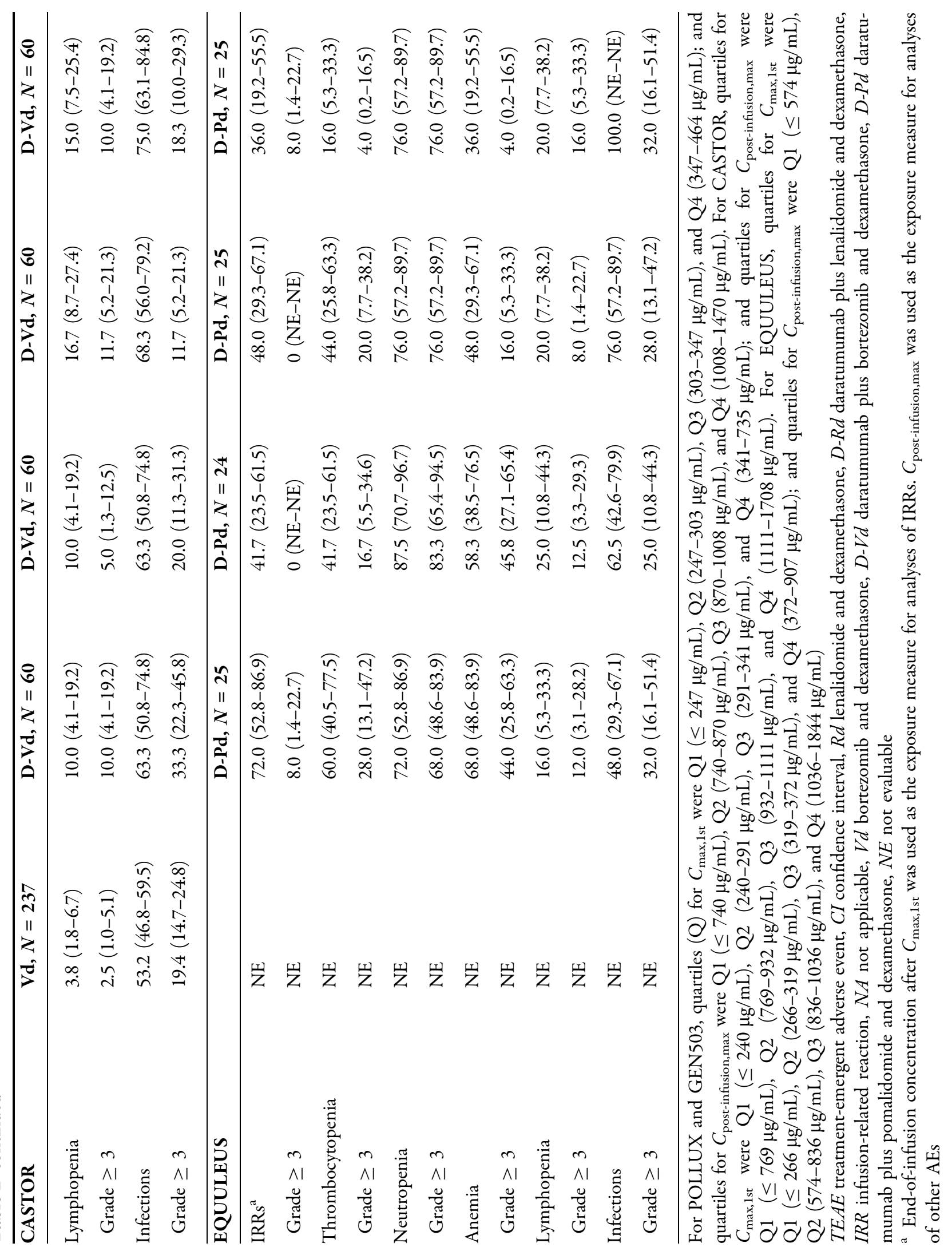




\section{Relationship Between Daratumumab Exposure and Safety}

There was no apparent relationship between $C_{\text {pre-infusion,max }}$ and any of the examined AEs of interest including thrombocytopenia, anemia, neutropenia, and lymphopenia based on data from the four combination therapy studies (Table 2). Although the rate of infections of any grade appeared to increase numerically with daratumumab exposure, this trend was not observed for grade 3 or higher infections. Similarly, no association between increasing $C_{\max , 1 \mathrm{st}}$ and the incidence of IRRs was identified. Across all of the studies, two patients were identified as being positive for anti-daratumumab antibodies, one from POLLUX and one from EQUULEUS; no differences between the PK of these patients and those without anti-daratumumab antibodies were discernable.

\section{DISCUSSION}

The benefits of adding daratumumab to combination regimens in the treatment of relapsed or relapsed and refractory $\mathrm{MM}$ necessitate an understanding of the PK profile of the drug in these regimens. This population PK analysis included data from a large number of subjects in early and late phase, multinational, clinical trials evaluating the use of daratumumab in combination therapy for patients with MM. These data allowed evaluation of important patient-related covariates across various patient populations and daratumumab dosing schedules as well as confirmation of the findings in monotherapy studies that had relatively smaller sample sizes [25].

The PK of daratumumab in combination with other treatment regimens were consistent with the PK of daratumumab administered as monotherapy [12-14].

Daratumumab exposure was similar when administered with various combination therapies and as monotherapy. The predicted $C_{\text {pre-infusion,max }}(632.8 \mu \mathrm{g} / \mathrm{mL}$ vs $530.65 \mu \mathrm{g} / \mathrm{mL}$, respectively) and mean half-life (23 vs 18 days, respectively) for daratumumab combination therapy versus monotherapy at the recommended dose and dosing schedule in POLLUX were similar. Achievement of steady state was consistently reached at approximately 5 months into the Q4W dosing period of the standard daratumumab dosing schedule. Moreover, daratumumab in combination with small-molecule therapies achieved greater than 99\% target saturation at trough concentrations in more than 90\% of patients following the $\mathrm{Q} 4 \mathrm{~W}$ dosing regimen. When the $C_{\text {pre-infusion,max }}$ was above the $\mathrm{EC}_{90}^{\mathrm{ORR}}$ identified at $274 \mu \mathrm{g} / \mathrm{mL}$ from the monotherapy studies [12], the ORR was markedly higher compared to those patients with $C_{\text {pre-infusion,max }}$ below $274 \mu \mathrm{g} / \mathrm{mL}$ (data not shown).

As observed in the monotherapy studies [14], no demographic or clinical characteristics were identified as having a clinically relevant effect on daratumumab PK. The covariate effects were all within $25 \%$, and thus, no dose adjustment is recommended on the basis of these covariates. Increasing body weight was associated with increased daratumumab clearance and volume of distribution; however, daratumumab exposures were consistent across patients' weight range, indicating that a body weight-based dose is reasonable and effective for administration of daratumumab in combination therapies. Elevated levels of IgG M-protein can lead to increased clearance of IgG-based monoclonal antibodies as a result of competition for the neonatal Fc receptor, which protects IgG from degradation [26]. Similar to findings observed in the daratumumab monotherapy studies [14], the IgG MM patients had lower concentrations of daratumumab than the non-IgG MM patients. However, the difference was only 23\%, approximately half of the magnitude of the difference observed in monotherapy studies [25], and was not considered clinically important.

Although most monoclonal antibodies have a biphasic PK profile with rapid distribution and slower elimination, individual PK properties of monoclonal antibodies are unique based on the biology of their target antigen [27]. The specific clearance of monoclonal antibodies is affected by binding to the target antigen, internalization, and subsequent intracellular protein catabolism. Fc-mediated effector functions not only contribute to the mechanism of action of monoclonal antibodies but also can impact their clearance. These factors highlight the need 
for PK evaluations of each therapeutic monoclonal antibody used for treatment of MM.

The exposure-efficacy analyses suggest that maximum clinical benefit on PFS has been attained for the majority of the subjects (approximately $75 \%$ ) with an acceptable safety profile at the recommended dose, $16 \mathrm{mg} / \mathrm{kg}$. Target saturation was maintained throughout dosing, even during Q4W dosing. At the recommended dose of $16 \mathrm{mg} / \mathrm{kg}$, the safety profile was acceptable, and there was no apparent relationship within the studied concentration range between drug exposure and IRRs, thrombocytopenia, anemia, neutropenia, and lymphopenia. The overall event rate of infection (any grade) appeared to increase with drug exposure, but this trend was not observed for grade 3 or higher infections.

There are some limitations to this study that are intrinsic to population PK analyses. Although a substantial number of MM patients from multiple clinical trials were utilized for analyses of PK over time, these analyses were limited by the schedule of PK sampling that varied slightly in each study protocol. In addition, data from clinical trials that utilized different daratumumab combination therapies were pooled for the analysis, with differences in sample size, phase of drug testing, and inclusion criteria between studies. Nevertheless, the PK data from this study are consistent with previous findings from daratumumab monotherapy studies and support the recommended $16 \mathrm{mg} / \mathrm{kg}$ dose of daratumumab in combination therapy.

\section{CONCLUSION}

These data support the recommended daratumumab $16 \mathrm{mg} / \mathrm{kg}$ dose in combination treatment regimens for patients with MM.

\section{ACKNOWLEDGEMENTS}

The authors thank the patients who participated in the POLLUX, CASTOR, GEN503, and EQUULEUS studies and their families, as well as the study co-investigators, research nurses, and coordinators at each of the clinical sites. The authors also thank Sam Laio, PhD, of Pharmax
Research, Inc, for his contributions to the data analysis of the study.

Funding. The clinical studies and the analyses presented here as well as Advances in Therapy's processing charges and Open Access fee were supported by research funding from Janssen Research \& Development, LLC. All authors had full access to all of the data in this study and take complete responsibility for the integrity of the data and accuracy of the data analysis.

Medical Writing and Other Editorial Assistance. Medical writing and editorial support were provided by Kristin Runkle, PhD, of MedErgy and funded by Janssen Global Services, LLC.

Authorship. All named authors meet the International Committee of Medical Journal Editors (ICMJE) criteria for authorship for this article, take responsibility for the integrity of the work as a whole, and have given their approval for this version to be published.

Disclosures. Xu Steven $\mathrm{Xu}$ is an employee of Janssen Research \& Development and owns stock in Johnson \& Johnson. Jordan Schecter is an employee of Janssen Research \& Development and owns stock in Johnson \& Johnson. Liping Zhang is an employee of Janssen Research \& Development and owns stock in Johnson \& Johnson. Yu-Nien Sun is an employee of Janssen Research \& Development and owns stock in Johnson \& Johnson. Pamela L. Clemens is an employee of Janssen Research \& Development and owns stock in Johnson \& Johnson. Richard Jansson owns stock in Johnson \& Johnson, is a former employee of Janssen and a current employee of Genmab US, Inc. Meletios A. Dimopoulos received consulting fees or honorarium from Amgen, Takeda, Janssen, and Celgene. Pieter Sonneveld received research funding from Janssen, Celgene, Amgen, Karyopharm, SkylineDx, Takeda, and Novartis; and received personal fees from Janssen, Celgene, and Amgen. P. Joy Ho received honorarium from Janssen-Cilag for an advisory board. Marcelo Capra served on a speakers bureau for Janssen, Amgen, and Roche. David 
Gomez received payment for lectures including service on speakers bureaus from Amgen, Celgene, and Janssen. Eva Medvedova is an employee of Oregon Health and Science University. Shinsuke Iida received consulting fees or honoraria from Janssen, Celgene, Ono Pharmaceutical, Novartis, Takeda, and BristolMyers Squibb; and received grants from Takeda, Ono Pharmaceutical, Kyowa-Hakko Kirin Co, Bristol-Myers Squibb, Celgene, Janssen, MSD Pharmaceuticals, Gilead, Daiichi Sankyo, Astellas Pharma, Toyama Chemical Co, Teijin Pharma Limited, Abbvie, and Sanofi. Andrew Belch, Merav Leiba, and Chang-Ki Min have nothing to disclose.

Compliance with Ethics Guidelines. All studies were conducted in accordance with the International Conference on Harmonisation Good Clinical Practice guidelines and the principles of the Declaration of Helsinki, and all patients provided written informed consent.

Data Availability. The data sharing policy of Janssen Pharmaceutical Companies of Johnson \& Johnson is available at https://www. janssen.com/clinical-trials/transparency. As noted on this site, requests for access to the study data can be submitted through Yale Open Data Access (YODA) Project site at http://yoda. yale.edu.

Open Access. This article is distributed under the terms of the Creative Commons Attribution-NonCommercial 4.0 International License (http://creativecommons.org/licenses/ by-nc/4.0/), which permits any noncommercial use, distribution, and reproduction in any medium, provided you give appropriate credit to the original author(s) and the source, provide a link to the Creative Commons license, and indicate if changes were made.

\section{REFERENCES}

1. Kumar SK, Lee JH, Lahuerta JJ, et al. Risk of progression and survival in multiple myeloma relapsing after therapy with IMiDs and bortezomib: a multicenter International Myeloma Working Group study. Leukemia. 2012;26(1):149-57.

2. Usmani S, Ahmadi T, Ng Y, et al. Analysis of realworld data on overall survival in multiple myeloma patients with $\geq 3$ prior lines of therapy including a proteasome inhibitor (PI) and an immunomodulatory drug (IMiD), or double refractory to a PI and an IMiD. Oncologist. 2016;21:1355-61.

3. de Weers M, Tai YT, van der Veer MS, et al. Daratumumab, a novel therapeutic human CD38 monoclonal antibody, induces killing of multiple myeloma and other hematological tumors. J Immunol. 2011;186(3):1840-8.

4. Krejcik J, Casneuf T, Nijhof IS, et al. Daratumumab depletes $\mathrm{CD}_{38}{ }^{+}$immune-regulatory cells, promotes $\mathrm{T}$-cell expansion, and skews T-cell repertoire in multiple myeloma. Blood. 2016;128(3):384-94.

5. Lammerts van Bueren J, Jakobs D, Kaldenhoven N, et al. Direct in vitro comparison of daratumumab with surrogate analogs of CD38 antibodies MOR03087, SAR650984 and Ab79. Blood. $2014 ; 124(21): 3474$.

6. Overdijk MB, Jansen JH, Nederend M, et al. The therapeutic CD38 monoclonal antibody daratumumab induces programmed cell death via Fcgamma receptor-mediated cross-linking. J Immunol. 2016;197(3):807-13.

7. Overdijk MB, Verploegen S, Bogels M, et al. Antibody-mediated phagocytosis contributes to the anti-tumor activity of the therapeutic antibody daratumumab in lymphoma and multiple myeloma. MAbs. 2015;7(2):311-21.

8. van de Donk NWCJ, Janmaat ML, Mutis T, et al. Monoclonal antibodies targeting CD38 in hematological malignancies and beyond. Immunol Rev. 2016;270(1):95-112.

9. Lokhorst HM, Plesner T, Laubach JP, et al. Targeting CD38 with daratumumab monotherapy in multiple myeloma. N Engl J Med. 2015;373(13):1207-19.

10. Lonial S, Weiss BM, Usmani S, et al. Daratumumab monotherapy in patients with treatment-refractory multiple myeloma (SIRIUS): an open-label, randomised, phase 2 trial. Lancet. 2016;387(10027):1551-60.

11. McKeage K. Daratumumab: first global approval. Drugs. 2016;76(2):275-81.

12. Xu XS, Yan X, Puchalski T, et al. Clinical implications of complex pharmacokinetics for daratumumab dose regimen in patients with relapsed/ refractory multiple myeloma. Clin Pharmacol Ther. 2016;101(6):721-4. 
13. Clemens PL, Yan X, Lokhorst HM, et al. Pharmacokinetics of daratumumab following intravenous infusion in relapsed or refractory multiple myeloma after prior proteasome inhibitor and immunomodulatory drug treatment. Clin Pharmacokinet. 2016;56(8):915-24.

14. Yan X, Clemens PL, Puchalski T, et al. Target-mediated drug disposition of daratumumab following intravenous infusion in relapsed or refractory multiple myeloma after prior proteasome inhibitors and immunomodulatory drugs: a population pharmacokinetic analysis. Blood. 2015;126(23):4222.

15. Usmani S, Weiss B, Bahlis NJ, et al. Clinical efficacy of daratumumab monotherapy in patients with heavily pretreated relapsed or refractory multiple myeloma. Blood. 2015;128(1):37-44.

16. Plesner T, Arkenau HT, Gimsing P, et al. Phase $1 / 2$ study of daratumumab, lenalidomide, and dexamethasone for relapsed multiple myeloma. Blood. 2016;128:1821-8.

17. Dimopoulos MA, Oriol A, Nahi H, et al. Daratumumab, lenalidomide, and dexamethasone for multiple myeloma. N Engl J Med. 2016;375(14):1319-31.

18. Palumbo A, Chanan-Khan A, Weisel K, et al. Daratumumab, bortezomib, and dexamethasone for multiple myeloma. N Engl J Med. 2016;375(8):754-66.

19. Bhatnagar V, Gormley NJ, Luo L, et al. FDA approval summary: daratumumab for treatment of multiple myeloma after one prior therapy. Oncologist. 2017;22(11):1347-53.
20. Chari A, Suvannasankha A, Fay JW, et al. Daratumumab plus pomalidomide and dexamethasone in relapsed and/or refractory multiple myeloma. Blood. 2017;130(8):974-81.

21. Mateos MV, Dimopoulos MA, Cavo M, et al. Daratumumab plus bortezomib, melphalan, and prednisone for untreated myeloma. $\mathrm{N}$ Engl J Med. 2018;378(6):518-28.

22. Guglieri-Lopez B, Perez-Pitarch A, Moes DJ, et al. Population pharmacokinetics of lenalidomide in multiple myeloma patients. Cancer Chemother Pharmacol. 2017;79(1):189-200.

23. Li Y, Xu Y, Liu L, Wang X, Palmisano M, Zhou S. Population pharmacokinetics of pomalidomide. J Clin Pharmacol. 2015;55(5):563-72.

24. LoRusso PM, Venkatakrishnan K, Ramanathan RK, et al. Pharmacokinetics and safety of bortezomib in patients with advanced malignancies and varying degrees of liver dysfunction: phase I NCI Organ Dysfunction Working Group Study NCI-6432. Clin Cancer Res. 2012;18(10):2954-63.

25. Yan X, Clemens PL, Puchalski T, et al. Influence of disease and patient characteristics on daratumumab exposure and clinical outcomes in relapsed or refractory multiple myeloma. Clin Pharmacokinet. 2018;57(4):529-38.

26. Roopenian DC, Akilesh S. FcRn: the neonatal Fc receptor comes of age. Nat Rev Immunol. 2007;7(9):715-25.

27. Kamath AV. Translational pharmacokinetics and pharmacodynamics of monoclonal antibodies. Drug Discov Today Technol. 2016;21-22:75-83. 